\title{
Genetic analysis of Pten and Tsc2 functional interactions in the mouse reveals asymmetrical haploinsufficiency in tumor suppression
}

\author{
Li Ma, ${ }^{1,2,3}$ Julie Teruya-Feldstein, ${ }^{2,6}$ Nille Behrendt, ${ }^{2,6}$ Zhenbang Chen, $^{1,2}$ Tetsuo Noda, ${ }^{4}$ \\ Okio Hino, ${ }^{5}$ Carlos Cordon-Cardo, ${ }^{2}$ and Pier Paolo Pandolfi ${ }^{1,2,7}$ \\ ${ }^{1}$ Cancer Biology and Genetics Program, ${ }^{2}$ Department of Pathology, Sloan-Kettering Institute, Memorial Sloan-Kettering \\ Cancer Center, New York, New York 10021, USA; ${ }^{3}$ Graduate Program in Physiology, Biophysics and Systems Biology, Weill \\ Graduate School of Medical Sciences, Cornell University, New York, New York 10021, USA; ${ }^{4}$ Department of Cell Biology \\ and ${ }^{5}$ Department of Experimental Pathology, Cancer Institute, Japanese Foundation for Cancer Research, \\ Tokyo 170-8455, Japan
}

The role of tumor suppressor haploinsufficiency in oncogenesis is still poorly understood. The PTEN and TSC2 tumor suppressors function to antagonize mTOR (mammalian target of rapamycin) activation by Akt; hence, compound heterozygous inactivation of Pten and $T s c 2$ in the mouse may in principle exacerbate the tumor phenotypes observed in the single mutants in a reciprocal manner. In contrast, we found that while Tsc2 heterozygosity unmasks Pten haploinsufficiency in growth and tumor suppression, tumorigenesis in $T s c 2^{+/-}$mutants is surprisingly not accelerated by Pten heterozygosity, even though mTOR activation is cooperatively enhanced by compound Pten/Tsc2 heterozygosity. We show that the wild-type alleles of both Pten and Tsc2 are retained in prostate tumors from both Pten $^{+/-}$and Pten ${ }^{+/-} \mathrm{Tsc2}^{+/-}$mice, whereas TSC-related tumor lesions are invariably associated with $T s c 2$ loss of heterozygosity $(\mathrm{LOH})$ in both $T s c 2^{+/-}$and Pten $^{+/-} \mathrm{Tsc2}^{+/-}$mice. These findings demonstrate that inactivation of TSC2 is epistatic to PTEN in the control of tumor initiation and progression and, importantly, that both Pten and Tsc2 are haploinsufficient for suppression of tumorigenesis initiated by Pten heterozygosity, while neither Pten nor Tsc2 is haploinsufficient for repression of carcinogenesis arising from $T s c 2$ heterozygosity, providing a rationale for the differential cancer susceptibility of the two human conditions associated with PTEN or TSC2 heterozygous mutations.

[Keywords: PTEN; TSC2; prostate cancer; haploinsufficiency]

Suplemental material is available at http://www.genesdev.org.

Received March 11, 2005; revised version accepted June 8, 2005.

Germline mutations in PTEN result in several syndromes characterized by hamartomas and high cancer susceptibility (Cantley and Neel 1999; Di Cristofano and Pandolfi 2000). PTEN alterations have been robustly implicated in human prostate cancer $(\mathrm{CaP})$, with PTEN deletions and/or mutations of at least one allele in up to $60 \%$ of primary prostate cancers, while homozygous PTEN inactivation is more frequently associated with metastatic prostate tissues (Gray et al. 1995; Cairns et al. 1997; Suzuki et al. 1998b). Tuberous sclerosis (TSC), an autosomal dominant tumor syndrome caused by mutations in TSC genes, is characterized by a wide spectrum

\footnotetext{
${ }^{6}$ These authors contributed equally to this work.

${ }^{7}$ Corresponding author.

E-MAIL p-pandolfi@ski.mskcc.org; FAX (212) 717-3102.

Article published online ahead of print. Article and publication date are

at http://www.genesdev.org/cgi/doi/10.1101/gad.1314405.
}

of hamartomas, but rare progression to malignancy with the exception of renal cancer (Gomez et al. 1999). Biochemical studies have demonstrated that the PTEN tumor suppressor gene encodes a phosphatase that dephosphorylates phosphatidylinositol 3,4,5-triphosphate (PIP3) and therefore antagonizes phosphatidylinositol 3-kinase (PI3K) activity (Cantley and Neel 1999; Di Cristofano and Pandolfi 2000), while mammalian TSC2 is an inhibitory phosphorylation substrate of Akt (Inoki et al. 2002; Manning et al. 2002). Genetic disruption of the Pten or Tsc2 gene in mice leads to tumorigenesis in distinct tissues. Pten ${ }^{+/-}$mice display hyperplastic-dysplastic features and are highly susceptible to developing epithelial tumors (Di Cristofano et al. 1998, 2001; Suzuki et al. 1998a; Podsypanina et al. 1999). These mice often die from a lethal autoimmune disorder, with a high penetrance of prominent lymphoadenopathy and splenomegaly showing marked lymphoid hyperplasia (Di Cris- 
tofano et al. 1999). In addition, Pten $^{+/-}$mice represent a faithful mouse model of $\mathrm{CaP}$, as they develop prostatic intraepithelial neoplasia (PIN) in the dorsolateral prostate (DLP) and the anterior prostate (AP), but not in the ventral prostate $(\mathrm{VP})$, which progresses to invasive $\mathrm{CaP}$ upon additional loss of the $C d k n 1 b$ gene (encoding the p2 $7^{\text {kip } 1}$ Cdk inhibitor) (Di Cristofano et al. 2001). On the other hand, Tsc2 $2^{+/-}$mice specifically develop renal carcinoma within a 6-10-mo latency and hepatic hemangioma with a later onset (12-18 mo; Kobayashi et al. 1999; Onda et al. 1999).

The recently identified biochemical relationship between mammalian TSC2 and Akt (Inoki et al. 2002; Manning et al. 2002) suggests that concomitant loss of PTEN and TSC2 may cooperate in tumorigenesis. However, the picture is further complicated by the negative feedback response resulting in impaired Akt activation which has been observed in Tsc2-deficient cells (Jaeschke et al. 2002). In addition, TSC2 appears to integrate signals from multiple pathways, including the Ras-Erk and AMPK pathways (Inoki et al. 2003; Ma et al. 2005). In this study, we focused on the PI3K pathway and sought to determine the genetic relationship between mammalian Pten and Tsc2 in tumor suppression by studying tumorigenesis in the $\mathrm{Pten}^{+/-} \mathrm{Tsc}^{+/-}$compound mouse mutants.

\section{Results}

Homozygous inactivation in either Pten or Tsc2 is embryonic lethal in mice (Di Cristofano et al. 1998; Suzuki et al. 1998a; Kobayashi et al. 1999; Onda et al. 1999;
Podsypanina et al. 1999). We therefore crossed $\mathrm{Pten}^{+/-}$ with Tsc2 $^{+/-}$mutants in an identical $129 \mathrm{~Sv} / \mathrm{C} 57 \mathrm{BL} / 6$ background. Four viable genotypes were obtained from this cross at approximately 1:1:1:1 ratio. Loss of one Tsc2 allele had no effect on survival on its own, but led to a significant reduction of lifespan in $\mathrm{Pten}^{+/-}$mice (Fig. 1A). Similar to Pten ${ }^{+/-}$mice, Pten ${ }^{+-} \mathrm{Tsc}^{+/-}$compound mutants often died from a severe lymphoadenopathy and splenomegaly. The $\mathrm{Pten}^{+/-}$and $\mathrm{Pten}^{+/-} \mathrm{TsC}^{+/-}$lymph nodes were indistinguishable in composition, as they both exhibited an expansion of B and T lymphocytes and appeared polymorphous in nature (Supplementary Fig. 1A). However, the lymph nodes from $\mathrm{Pten}^{+/-} \mathrm{Tsc}^{+/-}$ mice displayed increased staining of $\mathrm{Ki}-67$, a cell cyclespecific marker, compared with those from Pten $^{+/-}$mice (Fig. 1B), demonstrating that Tsc2 opposes the augmented cell proliferation triggered by Pten heterozygous loss. Although we did not observe an earlier onset of cervical lymphoadenopathy compared with $\mathrm{Pten}^{+/-}$ mice, several systemic signs distinctive of this disorder were exacerbated in the compound mutants: Acute inflammation, characterized by infiltration of polymorphonuclear cells, was observed in the liver of some of the compound mutants, but was never found in either single mutant (Supplementary Fig. 1B). Chronic inflammation, characterized by lymphoid infiltration, was observed in the kidney and liver with a $100 \%$ incidence in females older than $5 \mathrm{mo}$, and appeared much more severe than in Pten $^{+/-}$mice (Supplementary Fig. 1C). Additionally, the spleens in the majority of Pten $^{+/-} \mathrm{Tsc}^{+/-}$mice displayed red pulp expansion and a marked extramedullary hematopoiesis with megakarocytes and myeloid and erythroid elements, which was also massively exacerbated com-
Figure 1. Reduction of the Tsc2 dose leads to reduction of survival, enhanced lymphoid proliferation, and development of skin cancer in Pten $^{+/-}$mice. (A) KaplanMeier overall survival analysis shows a significant difference between $\mathrm{Pten}^{+/-} \mathrm{Tsc2}^{+/-}$and $\mathrm{Pten}^{+-}$mice $(P=0.03)$. (B, upper panel) Ki-67 staining reveals increased proliferation in the lymph node upon compound loss of Pten and Tsc2 (200x). A representative count in triplicate, out of three with similar results, is shown in the lower panel with standard deviations. $(C)$ Pathological features of a large skin lesion with squamous cell carcinoma in a 9-mo-old Pten $^{+/-} \mathrm{Tsc}^{+/-}$male mouse. (Panel $a$ ) The arrow points to an ulcerated skin lesion on the hind limb. (Panel $b$ ) Two detached squamous islands are indicated by the arrows (200x). (Panel c) A squamous pearl with central keratinization is shown (arrow; 200x). (Panel $d$ ) The arrow points at mitotic figures $(600 \times)$. (Panel e) Disorganized basal cells with atypia and dyskeratosis $(100 \times)$. (Panel f) A magnified image $(400 \times)$ of the boxed region in panel $e$. The solid arrow indicates nuclear atypia, and the open arrow indicates dyskeratosis.
A

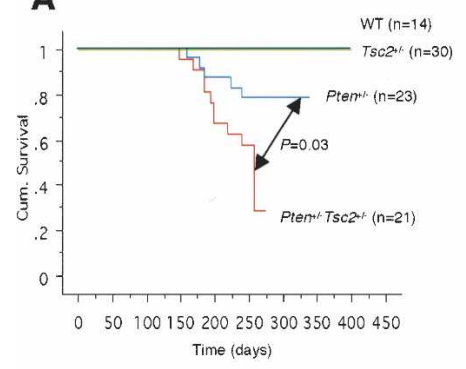

C

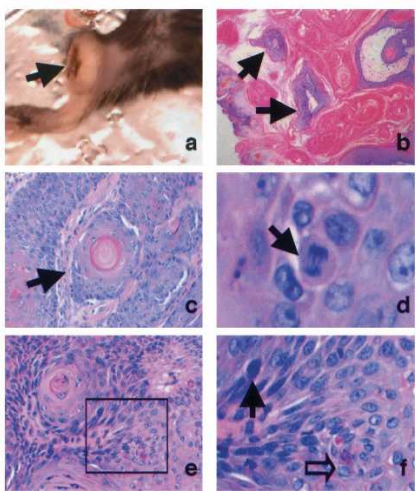

B
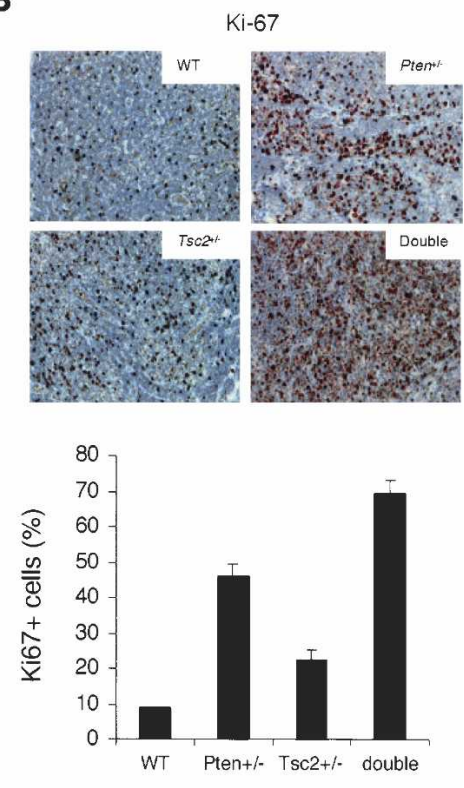
pared with Pten $^{+/-}$mutants (Supplementary Fig. 1D). Interestingly, associated with severe lymphoadenopathy and splenomegaly, $235 \%$ of $\mathrm{Pten}^{+/-} \mathrm{TsC2}^{+/-}$female mice (eight of 23) had ulcerated pelvic abscess filled with pus and necrotic cells, a phenotype never observed in the single mutants. Histological analysis revealed a necrotic center lined by neutrophils, foamy histiocytes, and a fibrotic wall (Supplementary Fig. 1E).

Loss of heterozygosity (LOH) and mutations of PTEN have been found in human patients with squamous cell carcinomas (SCC) (Shao et al. 1998). Pten complete inactivation in keratinocyte-specific Pten conditional mutants (k5Pten flox/flox mice) leads to skin tumorigenesis, as evidenced by the development of epidermal hyperplasia, squamous papillomas, and SCC (Suzuki et al. 2003). In contrast, Pten ${ }^{+/-}$mice do not develop skin malignancies, while SCC has been reported in $\mathrm{Pten}^{+/-}$Ink $4 \mathrm{a} / \mathrm{Arf}^{-/-}$ compound mutants (two of 46) (You et al. 2002). We observed that $\sim 10 \%$ of the Pten ${ }^{+-} \mathrm{Tsc}^{+/-}$double-heterozygous mutants (four of 41 ; with a 7-9-mo latency) showed large ulcerated SCCs, characterized by a number of typical features such as detached squamous islands surrounded by extensive keratinization, nests of squamous pearls with central keratinization, mitotic figures, disorganized basal cells with atypia, and dyskeratosis (Fig. 1C). Thus, compound Pten ${ }^{+/-} \mathrm{TsC}^{+/-}$mutants exhibit an expanded tumor spectrum.

Pten $^{+/-}$mice are highly prone to epithelial tumors in a variety of organs. However, some of the tumor types observed in Pten $^{+/-}$mutants do in fact occur after a long latency. For instance, PIN, which occurs at an $\sim 50 \%$ penetrance, is never observed prior to $8 \mathrm{mo}$ of age and never found to progress to $\mathrm{CaP}$ (Di Cristofano et al. 2001). Ssc2 $^{+/-}$mice were not susceptible to PIN or $\mathrm{CaP}$ (Fig. 2A,C). The majority of prostate glands in the $\mathrm{Tsc}^{+/-}$mutants analyzed were normal, with only a few glands becoming mildly hyperplastic in elder mutants (in mice older than $8 \mathrm{mo} ; n=4$; Fig. 2C). In contrast, Pten $^{+/-} \mathrm{Tsc}^{+/-}$compound mutants developed PIN at $100 \%$ penetrance, and more strikingly, CaP at a high penetrance. The onset of $\mathrm{CaP}$ in double-heterozygous males was $\sim 5$ mo of age, and by 9 mo virtually all males developed CaP (Fig. 2A,B). Moreover, these cancers exhibited manifest invasion, as evidenced by disruption of the basal membrane of the epithelium (Fig. 2C). By 8 mo of age, invasive $\mathrm{CaP}$ was observed in all three lobes (AP, $\mathrm{VP}$, and DLP), while the earliest occurrence of invasive $\mathrm{CaP}$ was observed in the AP of $\mathrm{Pten}^{+/-} \mathrm{Tsc}^{+/-}$compound mutants at 5 mo of age (Supplementary Fig. 2). Importantly, Pten immunostaining was retained in both normal tissues and neoplastic lesions in both Pten $^{+/-}$mice and Pten $^{+/-} \mathrm{TsC2}^{+/-}$compound mutants $(n=5$ mice and $>15$ neoplastic lesions examined for each genotype) (Fig. 2D).

Next, we performed laser capture microdissection of PIN cells from Pten ${ }^{+/-}$mice and prostate carcinoma cells from Pten $^{+/-} \mathrm{TsC2}^{+/-}$mice on paraffin-embedded sections, followed by LOH analysis of Pten and Tsc2. As shown in Figure 2E, the wild-type alleles of both genes were retained in prostate tumor cells in each case. These find-
A

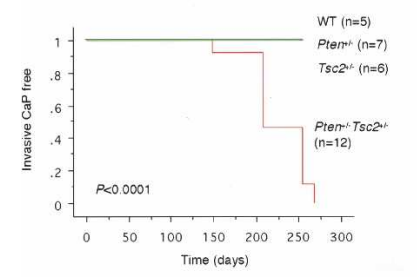

B

\begin{tabular}{lll}
\hline & Invasive CaP & PIN \\
\hline Pter $^{-T_{s c 2}+*}$ & $9 / 12(75 \%)$ & $12 / 12(100 \%)$ \\
Pten $^{*-}$ & $0 / 7$ & $4 / 7(57 \%)$ \\
\hline
\end{tabular}

C

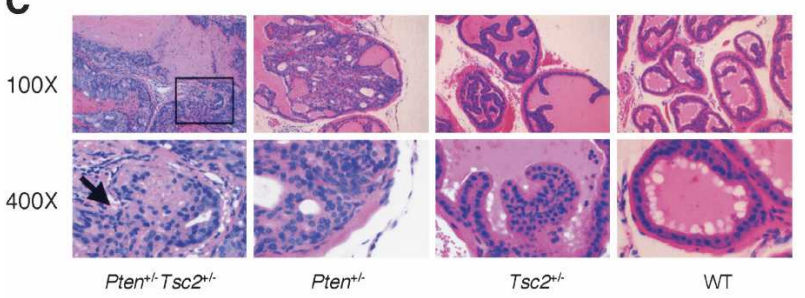

D

Pten

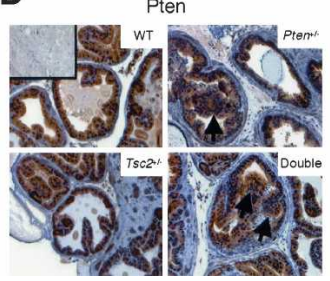

E

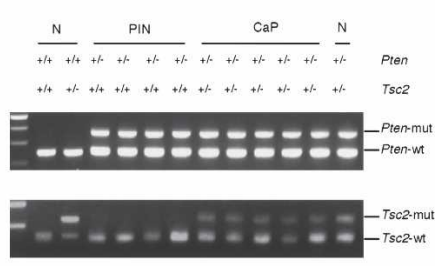

Figure 2. Reduction of the Tsc2 dose in $\mathrm{Pten}^{+/-}$mice leads to development of invasive $\mathrm{CaP}$, which retains both Pten and Tsc2 wild-type alleles. (A) Kaplan-Meier disease-free survival curve of invasive CaP. (B) Incidence of invasive CaP and PIN in Pten $^{+/-} \mathrm{Tsc2}^{+/-}$and Pten ${ }^{+/-}$mice. (C) Prostate tumorigenesis in AP of a 9-mo-old $\mathrm{Pten}^{+-} \mathrm{Tsc}^{+/-}$mouse and comparative analysis of littermates of various genotypes $(100 \times, 400 \times)$. The boxed region and arrow indicate the area of invasion. $(D)$ Expression of Pten in AP of 8-mo-old littermates (200x). Note the comparable level of Pten staining in neoplastic lesions (indicated by arrows) and in adjacent preneoplastic tissues. (Inset) Pten staining of a prostate from a Pten prostate conditional knockout mouse. $(E)$ LOH analysis of Pten and Tsc2 in prostate tumors. Genomic DNA was extracted from laser capture-microdissected prostate tumors and normal counterpart, and amplified by PCR to detect wild-type (wt) and mutated (mut) alleles of Pten and Tsc2, respectively. (N) Normal prostate cells.

ings provide strong support to the notion that Pten is haploinsufficient for suppression of pathogenesis of PIN and, importantly, that Tsc2 is haploinsufficient for repression of carcinogenesis initiated by Pten heterozygosity.

Unexpectedly, while tumorigenesis arising from Pten inactivation (e.g., prostate tumorigenesis) was markedly accelerated by Tsc2 heterozygosity, the tumor phenotypes specifically observed in $T s c 2^{+/-}$mice were not enhanced in the compound mutants. As seen in the Eker rat model of TSC, $\mathrm{TSC}^{+/-}$mice were previously reported to display renal cystadenomas and/or renal carcinomas (in human TSC patients, angiomyolipomas are more common renal lesions than renal carcinomas, suggesting species-specific phenotypes), accompanied by frequent loss of the wild-type Tsc2 allele (Kobayashi et al. 1999; 
Onda et al. 1999). Consistent with these reports, $\mathrm{Tsc2}^{+/-}$mice developed renal cystadenomas and/or renal carcinomas. However, $\mathrm{Tsc}^{+/-}$and $\mathrm{Pten}^{+-} \mathrm{Tsc2}^{+/-} \mathrm{mu}-$ tants developed these tumors with similar onset and incidence (Fig. 3A-C). Kidneys from both $\mathrm{Tsc}^{+/-}$and Pten $^{+/-} \mathrm{TsC2}^{+/-}$mice often showed macroscopically visible cysts on the surface. The cysts could be simple cysts, but mostly were associated with adenomas or carcinomas in the cortical region. Conversely, renal carcinomas could be cystic or unassociated with cysts, with the majority displaying a papillary feature (Fig. 3D). The other tumor phenotype observed in $\mathrm{Tsc}^{+/-}$mice in the mixed 129Sv/C57BL/6 background was unexpected hepatic hemangiomas after a long latency (12-18 mo), which are not seen in either TSC patients or the Eker rat model of TSC (Kobayashi et al. 1999). No hepatic hemangiomas were found in a 12-mo follow-up in either $\mathrm{Tsc2}^{+/-}$or $\mathrm{Pten}^{+/-} \mathrm{Tsc2}^{+/-}$mice in the genetic background we studied.

To explain why renal carcinogenesis in $T s c 2^{+/-}$mice is not accelerated upon Pten heterozygous loss, two possibilities can be entertained: (1) The tumor-promoting effect of Pten deficiency might be counterbalanced by less frequent Tsc2 LOH in the compound mutant; (2) more likely, tumorigenesis initiated by Tsc2 heterozygosity must be followed by Tsc2 $\mathrm{LOH}$ in order to give rise to full-blown malignancy. To investigate this latter possibility, we performed $\mathrm{LOH}$ analysis on microdissected renal carcinomas from both $\mathrm{Tsc}^{+/-}$mice and Pten $^{+/-} \mathrm{Tsc}^{+/-}$compound mutants. While no Pten LOH was detected, the wild-type Tsc2 allele was found invariably lost in all renal cancers from these mice (Fig. 3E). Thus, in contrast to what was observed in the prostate, Tsc2 is not haploinsufficient for repression of renal carcinogenesis even in a Pten $^{+/-}$genetic background.
Next, we sought to determine the biochemical and biological consequences of concomitant heterozygous loss of Pten and Tsc2, both in vivo as well as in primary mouse embryonic fibroblasts (MEFs). As predicted by the specific role of Pten in the PI3-kinase pathway, the phospho-Akt level was found increased in the prostate of either Pten ${ }^{+/-}$or Pten ${ }^{+/-}$Tsc2 $^{+/-}$mice ( $n=4$ mice examined for each genotype; representative results shown in Fig. 4A). In contrast, phosphorylation at S2448 of the key regulator of mRNA translation mTOR (mammalian target of rapamycin; phosphorylation at this residue occurs via activation of the PI3K/Akt pathway, and can be suppressed by TSC1-TSC2 overexpression and augmented by TSC2 RNA interference [RNAi]) (Inoki et al. 2002) was found markedly enhanced only in the prostates (in both normal epithelium and carcinomas) of Pten $^{+/-} \mathrm{TsC2}^{+/-}$mice $(n=4$ mice examined for each genotype; representative results shown in Fig. 4B). To date, it remains in question whether mTOR S2448 phosphorylation always leads to increased downstream signaling (Hay and Sonenberg 2004). We therefore examined the phospho-S6 level in prostate homogenates from three to five 6-mo-old mice of each genotype by immunoblot analysis (representative results shown in Fig. 4C), and found that signaling downstream of mTOR was also manifestly enhanced in all $\mathrm{Pten}^{+/-} \mathrm{Tsc}^{+/-}$prostate tissues. These results are therefore in agreement with the fact that TSC2 functions downstream of Akt and upstream of TOR (Potter et al. 2001; Tapon et al. 2001). In Pten $^{+/-}$mice, expression of the Ki-67 proliferation marker was mostly found increased in the PIN lesions, whereas in double-heterozygous mice a marked increase in the proliferative index was observed not only in the tumor lesions, but also in the preneoplastic tissues (Fig. 4D).
Figure 3. Renal carcinogenesis in $\mathrm{Pten}^{+/-} \mathrm{Tsc2}^{+/-}$mice is associated with Tsc2 LOH and is not accelerated by reduction of the Pten dose. (A) Kaplan-Meier disease-free survival curve of renal cystadenoma. (B) Kaplan-Meier disease-free survival curve of renal adenocarcinoma. $(C)$ Incidence of renal cystadenoma and renal carcinoma in Pten $^{+/-} \mathrm{Tsc}^{+/-}$and Tsc2 ${ }^{+/-}$mice. (D) H\&E sections show a papillary carcinoma in the kidney of a 5-mo-old Pten $^{+/-} \mathrm{Tsc2}^{+/-}$mouse, and a papillary cystic carcinoma in the kidney of a Tsc2 $2^{+/-}$littermate $(40 \times, 400 \times)$. (E) LOH analysis of Pten and Tsc2 in renal carcinomas. Genomic DNA was extracted from laser capture-microdissected renal carcinomas and a normal counterpart, and amplified by PCR to detect wild-type (wt) and mutated (mut) alleles of Pten and Tsc2, respectively. (RC) Renal carcinoma; (N) normal kidney cells; (-) no template.
A

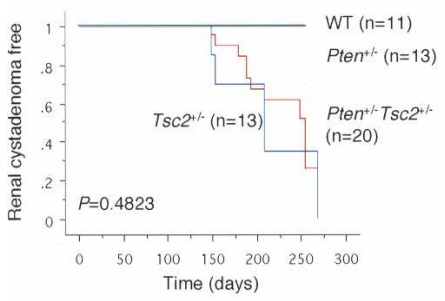

B

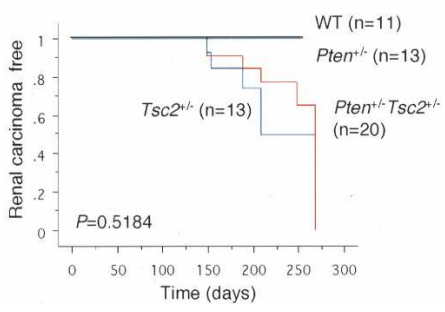

C

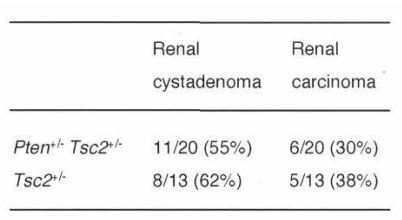

D

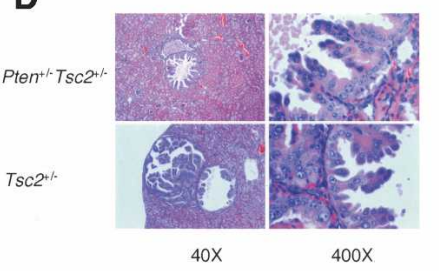

E $\frac{\mathrm{RC}}{+1++1++1-+1 /++1+} \frac{\mathrm{N}}{\text { Pten }}$

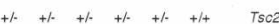

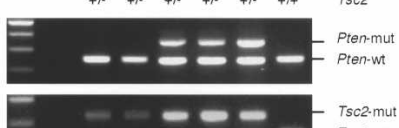

$-\infty=-T_{T s c 2 \cdot \mathrm{m}}$ 
A

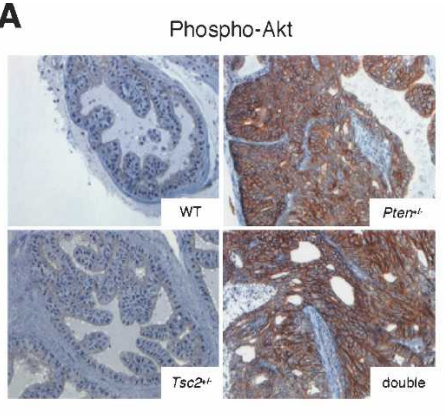

C

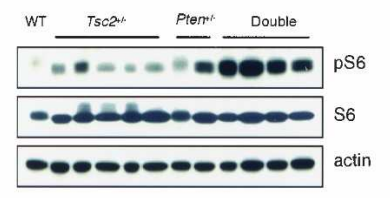

D

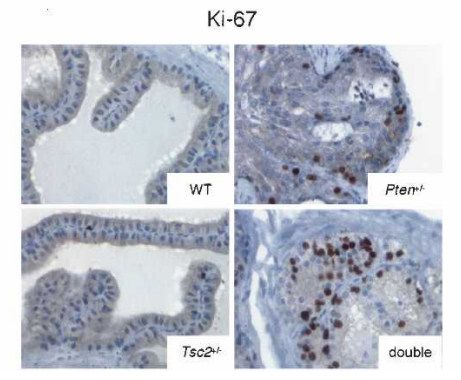

B
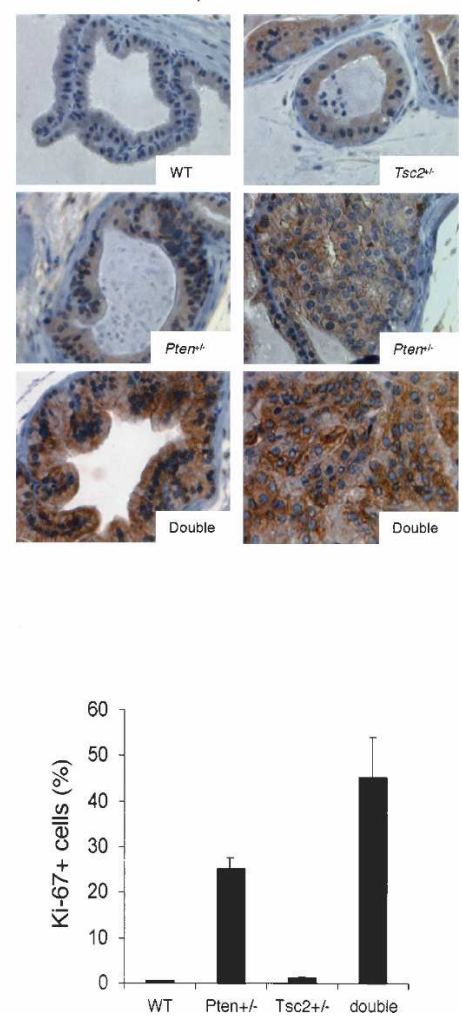

Figure 4. Biochemical and biological effects of compound loss of Pten and Tsc2 in the prostate. (A) Marked increase in phospho-Akt level in the prostates of either Pten ${ }^{+/}$or Pten ${ }^{+/}$Tsc2 $^{+/-}$mice (200×). (B) PhosphomTOR level is markedly increased only in the prostates of Pten $^{+/-} \mathrm{Tsc2}^{+/-}$mice (200×). A PIN lesion from a Pten ${ }^{+/-}$ mouse and a prostate carcinoma from a $\mathrm{Pten}^{+/-} \mathrm{Tsc2}^{+/-}$ mouse are shown along with normal epithelium from all genotypes. $(C)$ Western blot analysis of prostate homogenates of various genotypes shows a marked increase in phospho-S6 level in the prostate of $\mathrm{Pten}^{+/-} \mathrm{Tsc2}^{+/-}$mice. (D, left panel) Ki-67 staining reveals increased proliferation in the prostate upon compound loss of Pten and Tsc2 $(400 \times)$. Note that the area with prominent Ki-67 signals in the prostate of $\mathrm{Pten}^{+/-} \mathrm{Tsc}^{+/-}$mice is preneoplastic, while the area shown in the prostate of $\mathrm{Pten}^{+/-}$mice is a PIN lesion. A representative count in triplicate out of three with similar results is shown in the right panel with standard deviations.
In MEFs at early passages, both $\mathrm{Pten}^{+/-}$and $\mathrm{Pten}^{+/-} \mathrm{TsC}^{+/-}$ cells showed a more rapid proliferation rate than wild-type cells (Fig. 5A). At late passages, however, wild-type MEFs exhibited pronounced cellular senescence (as scored by senescence-associated [SA]- $\beta$-gal staining), which could be almost completely overcome by compound Pten and Tsc2 heterozygous inactivation, whereas only a partial reduction in cellular senescence was observed in MEFs from single mutants (Fig. 5B).

We observed that Pten and Tsc2 heterozygous inactivation led to diminished expression level of both proteins while compared with wild-type cells, the Akt phosphorylation level was reduced in $\mathrm{Tsc}^{+/-}$MEFs but increased in both $\mathrm{Pten}^{+/-}$and $\mathrm{Pten}^{+/-} \mathrm{Tsc}^{+/-} \mathrm{MEFs}$ (Fig. 5C), indicating that the feedback inhibition of PI3KAkt triggered by Tsc2 deficiency can be overcome by loss of Pten. We also examined the status of the two main known direct substrates of mTOR, the p70S6 kinase $(\mathrm{S} 6 \mathrm{~K})$ and 4E-BP1. When phosphorylated by $\mathrm{mTOR}, \mathrm{S} 6 \mathrm{~K}$ and 4E-BP1 can promote protein synthesis (Gingras et al. 2001). We found that phosphorylation of S6K and 4E-BP1 at the mTOR-dependent site was comparable to that of wild-type cells /or only slightly increased) in both Pten $^{+/-}$and $\mathrm{Tsc}^{+/-}$single-heterozygous MEFs. In contrast, compound inactivation of Pten and Tsc2 led to enhanced S6K and 4E-BP1 phosphorylation (Fig. 5C), further demonstrating that concomitant loss of Pten and Tsc2 has a cooperative effect in the activation of mTOR.

\section{Discussion}

Our data conclusively demonstrate that Pten and Tsc2 cooperatively antagonize the activation of $\mathrm{mTOR}$ in vivo and act synergistically in tumor suppression. In turn, these findings underscore the relevance of TOR activation and blockade in cancer pathogenesis and therapy, respectively, in tumors driven by heterozygous PTEN inactivation. However, the relationship between Pten and Tsc2 and the outcome of their heterozygous inactivation is surprisingly not reciprocal (Fig. 5D): Tsc2 does contribute to prevention of specific tumors caused by Pten heterozygous inactivation, i.e., tumorigenesis arising from Pten heterozygosity can progress to full-blown cancer upon partial loss of Tsc2, without the need to lose the wild-type Pten allele, whereas Tsc2 LOH is an indispensable step for tumors initiated by Tsc2 partial deficiency to become malignant. Thus, while Tsc2 appears to be epistatic to Pten in tumor suppression in vivo, in agreement with the hierarchical relationships of these two tumor suppressors in the PI3K signal transduction pathway in vitro, our analyses demonstrate that in the presence of compound heterozygosity, both Pten and Tsc2 are haploinsufficient for suppression of tumorigenesis initiated by Pten heterozygosity, and that neither Pten nor Tsc2 is haploinsufficient for repression of carcinogenesis arising from Tsc2 heterozygosity. This is in complete agreement with what is reported by Manning et al. (2005). They also report that the development of renal cystadenomas and/or renal carcinomas is not accelerated 
A

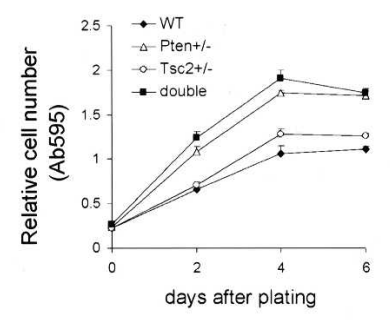

B

C

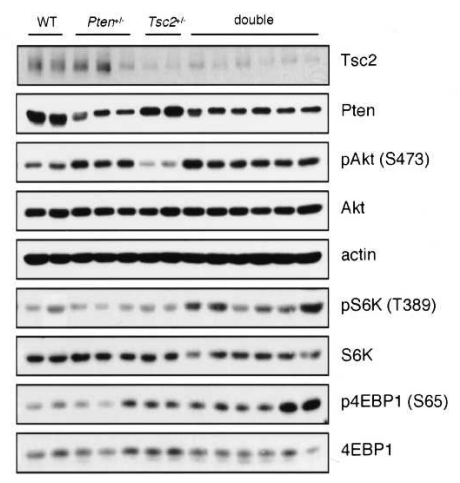

Figure 5. Biochemical and biological effects of compound loss of Pten and Tsc2 in MEFs. (A) Growth curves of MEFs at passage 3 show a growth advantage of $\mathrm{Pten}^{+/-} \mathrm{Tsc}^{+/-}$and $\mathrm{Pten}^{+/-}$MEFs over either $T s c 2^{+/-}$or wild-type MEFs. A representative experiment in triplicate out of three with similar results is shown with standard deviations. (B) SA- $\beta$-gal staining of MEFs at passage 10 shows bypass of cellular senescence in $\mathrm{Pten}^{+/-} \mathrm{Tsc2}^{+/-}$ MEFs. A representative experiment in triplicate out of three with similar results is shown with standard deviations. $(C)$ Western blot analysis of Tsc2, Pten, phospho-Akt, phosphoS6K, and phospho-4EBP1 levels in primary MEFs of various genotypes. (D) Model of asymmetrical haploinsufficiency and the nonreciprocal relationship between Pten and Tsc2 in tumor suppression. While tumorigenesis initiated by Pten heterozygous inactivation (e.g., PIN) undergoes progression to cancer upon either Pten LOH or partial loss of another tumor suppressor gene (e.g., Tsc2, or $C d k n 1 b$ encoding the p $27^{\text {kip } 1}$ protein), TSC-associated tumor lesions progress to full-blown malignancy only upon complete loss of the Tsc2 gene.

in Pten $^{+/-} \mathrm{Tsc}^{+/-}$mutants. Furthermore, even though hepatic hemangiomatosis displays an earlier onset in Pten $^{+/-} \mathrm{TsC2}^{+/-}$compound mice than in single $\mathrm{Tsc}^{+/-}$ mutants in their genetic background, Manning et al. (2005) report that these tumors are nevertheless invariably characterized by loss of Tsc2 (but not Pten) expression, consistent with the frequent Tsc2 LOH previously reported in these tumors from Tsc2 single-heterozygous mice. In contrast, they find no Pten LOH in the hyperplastic lymph nodes from either $\mathrm{Pten}^{+/-}$or Pten $^{+/-}$Tsc2 $^{+/-}$mice (Manning et al. 2005). These results further reinforce our finding of both asymmetrical haploinsufficiency and a nonreciprocal relationship between Pten and Tsc2. The possible underlying mechanisms for this enhanced phenotype that Manning et al. (2005) report could be accelerated Tsc2 LOH induced by heterozygous loss of Pten in hepatic tumors in their genetic

background and/or, as Manning et al. propose, reactivation of pathways downstream of Akt other than the mTOR pathway.

Taken together, our findings could explain the overall low incidence of malignant lesions in TSC patients compared with the higher incidence observed in tumorigenesis associated with PTEN heterozygous mutations (Fig. 5D). More generally, our data lend further support to the notion that combinatorial tumor suppressor haploinsufficiency is of critical importance in cancer pathogenesis.

It should be noted that loss of Tsc1 or Tsc2 was recently found to result in impaired PI3K-Akt activation (Jaeschke et al. 2002; Zhang et al. 2003). On the basis of this observation, one might predict that the phenotype of Pten $^{+/-}$mice would be suppressed rather than accelerated by loss of Tsc2. However, this negative feedback loop has been shown to inhibit the PI3K-Akt pathway by down-regulating upstream activators of PI3K such as PDGF receptor and insulin receptor substrate (IRS) proteins (Zhang et al. 2003; Harrington et al. 2004; Shah et al. 2004). Upon Pten deficiency, PIP3 accumulates and Akt is activated even in the absence of receptor tyrosine kinase signaling. Indeed, we demonstrate here that in the compound mutants, heterozygous Pten inactivation is sufficient to overcome the negative feedback and activate Akt in vivo (Fig. 4A) and in MEFs (Fig. 5C), while accompanying loss of Tsc2 leads to elevated mTOR activation (Figs. 4B,C, 5C). Therefore, concomitant reduction in the dosage of two tumor suppressors acting in the same pathway, which gives rise to enhanced signaling through this pathway, can explain the accelerated phenotype. This, in turn, has important therapeutic implications, as based on our report that combination treatment with agents that concomitantly block PI3-kinase/ Akt and mTOR activation could be beneficial. Furthermore, our findings prompt the analysis of the status of the TSC complex/genes in human cancers that suffer from PTEN functional loss (e.g., prostate cancer).

\section{Materials and methods}

Mating, genotyping, and follow-up of mice

Pten $^{+/-}$or $\mathrm{Tsc2}^{+/-}$single-heterozygous mice in the identical 129Sv/C57BL/6 background were crossed to obtain Pten $^{+/-}$Tsc2 $^{+/-}$ compound mutants. Genotyping by PCR on tail DNA was performed as previously described (Di Cristofano et al. 1998; Kobayashi et al. 1999). All mice from the above cross were divided into two groups. One group of mice was monitored throughout their lives to determine the overall survival. In a second group, two to four mice (males and females) from each genotype were sacrificed every month between 4 and 9 mo for gross and microscopic examination of all organs - the prostate, kidney, and liver in particular-to determine the disease onset and incidence.

\section{Autopsy, histopathology, and immunohistochemistry}

Mice were autopsied and all tissues were analyzed regardless of their pathological condition. Tissue samples were fixed in $10 \%$ buffered formalin for $12 \mathrm{~h}$, followed by wash with PBS and trans- 
fer to $70 \%$ ethanol, and the following histological procedures (paraffin embedding, sectioning, and hematoxylin and eosin $[\mathrm{H} \& \mathrm{E}]$ staining) were provided by Histoserv, Inc. The IHC detection using anti-B220 (Pharmingen), anti-CD3 (Dako), antiPTEN (Neomarkers), anti-phospho-Akt and anti-phosphomTOR (Cell Signaling Technology), and anti-Ki-67 (Novocastra Laboratories Ltd.) was performed with automated IHC procedures at the Molecular Cytology Core Facility of Memorial Sloan-Kettering Cancer Center.

\section{Laser capture microdissection and DNA extraction}

Formalin-fixed, paraffin-embedded blocks were cut into 7- $\mu \mathrm{m}$ thick sections on uncharged glass slides. Sections were slightly stained with hematoxylin and microdissected using a PALM laser microdissection and pressure catapulting (LMPC) system. A $0.5-\mathrm{mL}$ clean microcentrifuge tube was inserted in the tube holder with $20 \mu \mathrm{L}$ nuclease-free water in the cap. The marked cells were catapulted into the cap. The cap was then closed and the tube was centrifuged at 13,000 rpm for 5-10 min. Twenty microliters of ATL buffer (a component of QIAamp DNA Micro Kit; QIAGEN) and $10 \mu \mathrm{L}$ of Proteinase K solution $(20 \mathrm{mg} / \mathrm{mL})$ were added. The samples were vortexed for 10-15 sec, followed by incubation for $20-24 \mathrm{~h}$ at $55^{\circ} \mathrm{C}$ and then for $10 \mathrm{~min}$ at $95^{\circ} \mathrm{C}$. The samples were then processed for DNA extraction using the QIAamp DNA Micro Kit, according to the manufacturer's instructions.

\section{LOH analysis}

Genomic DNA from formalin-fixed, paraffin-embedded tissues was prepared as described above. Primers used for Pten LOH analysis were P1 (5'-TGGGAAGAACCTAGCTTGGAGG-3' in the intron 5 region), P2 (5'-TTCCATTTGTCACGTCCTG CAC-3' in the neo region), and P3 (5'-ACTCTACCAGCCCA AGGCC-3' in the intron 5 region). P1 and P3 were used for the wild-type allele, and P2 and P3 were used for the mutated allele. Primers used for Tsc2 LOH analysis and PCR conditions were previously described (Kobayashi et al. 1999).

\section{MEF isolation, growth, and senescence}

MEFs were derived from 13.5-d-old embryos using a previously described protocol (Kamijo et al. 1997) and maintained in Dulbecco's modified Eagle medium (DMEM) supplemented with $10 \%$ fetal bovine serum (FBS), 2 mM glutamine, and $100 \mathrm{U} / \mathrm{mL}$ penicillin and streptomycin (GIBCO). Growth curves were determined using a crystal violet method as previously described (Serrano et al. 1997). SA- $\beta$-gal staining was performed using the Senescence Detection Kit (Oncogene) according to the manufacturer's instructions.

\section{Protein analysis}

Prostates from dissected mice were minced and incubated in RIPA lysis buffer (150 mM NaCl, $10 \mathrm{mM}$ Tris at $\mathrm{pH} 7.5,1 \%$ NP40, $1 \%$ deoxycholate, $0.1 \%$ SDS, protease inhibitor cocktail [Roche], phosphatase inhibitor cocktail [Sigma]), and homogenized using a tissue homogenizer. MEF cells were harvested and directly lysed in RIPA lysis buffer. Proteins from total lysates were resolved by SDS-PAGE, transferred to a nitrocellulose membrane, blocked in 5\% nonfat milk or BSA, and blotted with the following antibodies: anti-PTEN (Cascade Bioscience), anti-TSC2 (Santa Cruz), anti-actin (Sigma), anti-phospho-pAkt (S473), anti-Akt, anti-phospho-S6K (T389), anti-S6K, anti-phos-
pho-4EBP1 (S65), anti-4EBP1, anti-phospho-S6 (S235/S236), and anti-S6 (Cell Signaling Technology).

\section{Acknowledgments}

We thank Achim Jungbluth for kindly providing access to the PALM LMPC system, and Katia Manova, Craig Farrell, and Tabita Beharry for assistance with the automated IHC. We are grateful to the members of the Pandolfi lab for critical discussion, and to Linda DiSantis for editing the manuscript. We also thank Brendan Manning and Lewis Cantley for kindly sharing unpublished information. This study was supported in part by NCI grants RO1 CA-82328, U01 CA-84292 and by the I.T. Hirschl/M. Weill-Caulier Foundation to P.P.P and C.C-C.

\section{References}

Cairns, P., Okami, K., Halachmi, S., Halachmi, N., Esteller, M., Herman, J.G., Jen, J., Isaacs, W.B., Bova, G.S., and Sidransky, D. 1997. Frequent inactivation of PTEN/MMAC1 in primary prostate cancer. Cancer Res. 57: 4997-5000.

Cantley, L.C. and Neel, B.G. 1999. New insights into tumor suppression: PTEN suppresses tumor formation by restraining the phosphoinositide 3-kinase/AKT pathway. Proc. Natl. Acad. Sci. 96: 4240-4245.

Di Cristofano, A. and Pandolfi, P.P. 2000. The multiple roles of PTEN in tumor suppression. Cell 100: 387-390.

Di Cristofano, A., Pesce, B., Cordon-Cardo, C., and Pandolfi, P.P. 1998. Pten is essential for embryonic development and tumour suppression. Nat. Genet. 19: 348-355.

Di Cristofano, A., Kotsi, P., Peng, Y.F., Cordon-Cardo, C., Elkon, K.B., and Pandolfi, P.P. 1999. Impaired Fas response and autoimmunity in Pten $^{+/-}$mice. Science 285: 2122-2125.

Di Cristofano, A., De Acetis, M., Koff, A., Cordon-Cardo, C., and Pandolfi, P.P. 2001. Pten and p27KIP1 cooperate in prostate cancer tumor suppression in the mouse. Nat. Genet. 27: 222-224.

Gingras, A.C., Raught, B., and Sonenberg, N. 2001. Regulation of translation initiation by FRAP/mTOR. Genes \& Dev. 15: 807-826.

Gomez, M.R., Sampson, J.R., and Whittemore, V.H. 1999. Tuberous sclerosis complex. Oxford University Press, New York.

Gray, I.C., Phillips, S.M., Lee, S.J., Neoptolemos, J.P., Weissenbach, J., and Spurr, N.K. 1995. Loss of the chromosomal region 10q23-25 in prostate cancer. Cancer Res. 55: 48004803.

Harrington, L.S., Findlay, G.M., Gray, A., Tolkacheva, T., Wigfield, S., Rebholz, H., Barnett, J., Leslie, N.R., Cheng, S., Shepherd, P.R., et al. 2004. The TSC1-2 tumor suppressor controls insulin-PI3K signaling via regulation of IRS proteins. J. Cell Biol. 166: 213-223.

Hay, N. and Sonenberg, N. 2004. Upstream and downstream of mTOR. Genes \& Dev. 18: 1926-1945.

Inoki, K., Li, Y., Zhu, T., Wu, J., and Guan, K.L. 2002. TSC2 is phosphorylated and inhibited by Akt and suppresses mTOR signalling. Nat. Cell Biol. 4: 648-657.

Inoki, K., Zhu, T., and Guan, K.L. 2003. TSC2 mediates cellular energy response to control cell growth and survival. Cell 115: 577-590.

Jaeschke, A., Hartkamp, J., Saitoh, M., Roworth, W., Nobukuni, T., Hodges, A., Sampson, J., Thomas, G., and Lamb, R. 2002. Tuberous sclerosis complex tumor suppressor-mediated S6 kinase inhibition by phosphatidylinositide-3-OH kinase is 
Ma et al.

mTOR independent. J. Cell Biol. 159: 217-224.

Kamijo, T., Zindy, F., Roussel, M.F., Quelle, D.E., Downing, J.R., Ashmun, R.A., Grosveld, G., and Sherr, C.J. 1997. Tumor suppression at the mouse INK4a locus mediated by the alternative reading frame product p19ARF. Cell 91: 649-659.

Kobayashi, T., Minowa, O., Kuno, J., Mitani, H., Hino, O., and Noda, T. 1999. Renal carcinogenesis, hepatic hemangiomatosis, and embryonic lethality caused by a germ-line Tsc2 mutation in mice. Cancer Res. 59: 1206-1211.

Ma, L., Chen, Z., Erdjument-Bromage, H., Tempst, P., and Pandolfi, P.P. 2005. Phosphorylation and functional inactivation of TSC2 by Erk implications for tuberous sclerosis and cancer pathogenesis. Cell 121: 179-193.

Manning, B.D., Tee, A.R., Logsdon, M.N., Blenis, J., and Cantley, L.C. 2002. Identification of the tuberous sclerosis complex-2 tumor suppressor gene product tuberin as a target of the phosphoinositide 3-kinase/akt pathway. Mol. Cell 10: 151-162.

Manning, B.D., Logsdon, M.N., Lipovsky, A., Abbott, D., Kwiatkowski, D.J., and Cantley, L.C. 2005. Feedback inhibition of Akt signaling limits the growth of tumors lacking Tsc2. Genes \& Dev. (this issue).

Onda, H., Lueck, A., Marks, P.W., Warren, H.B., and Kwiatkowski, D.J. 1999. Tsc2 ${ }^{+/-}$mice develop tumors in multiple sites that express gelsolin and are influenced by genetic background. J. Clin. Invest. 104: 687-695.

Podsypanina, K., Ellenson, L.H., Nemes, A., Gu, J., Tamura, M., Yamada, K.M., Cordon-Cardo, C., Catoretti, G., Fisher, P.E., and Parsons, R. 1999. Mutation of Pten/Mmacl in mice causes neoplasia in multiple organ systems. Proc. Natl. Acad. Sci. 96: 1563-1568.

Potter, C.J., Huang, H., and Xu, T. 2001. Drosophila Tsc1 functions with Tsc2 to antagonize insulin signaling in regulating cell growth, cell proliferation, and organ size. Cell 105: 357368.

Serrano, M., Lin, A.W., McCurrach, M.E., Beach, D., and Lowe, S.W. 1997. Oncogenic ras provokes premature cell senescence associated with accumulation of p53 and p16INK4a. Cell 88: 593-602.

Shah, O.J., Wang, Z., and Hunter, T. 2004. Inappropriate activation of the TSC/Rheb/mTOR/S6K cassette induces IRS1/2 depletion, insulin resistance, and cell survival deficiencies. Curr. Biol. 14: 1650-1656.

Shao, X., Tandon, R., Samara, G., Kanki, H., Yano, H., Close, L.G., Parsons, R., and Sato, T. 1998. Mutational analysis of the PTEN gene in head and neck squamous cell carcinoma. Int. J. Cancer 77: 684-688.

Suzuki, A., de la Pompa, J.L., Stambolic, V., Elia, A.J., Sasaki, T., del Barco Barrantes, I., Ho, A., Wakeham, A., Itie, A., Khoo, W., et al. 1998a. High cancer susceptibility and embryonic lethality associated with mutation of the PTEN tumor suppressor gene in mice. Curr. Biol. 8: 1169-1178.

Suzuki, H., Freije, D., Nusskern, D.R., Okami, K., Cairns, P., Sidransky, D., Isaacs, W.B., and Bova, G.S. 1998b. Interfocal heterogeneity of PTEN/MMAC1 gene alterations in multiple metastatic prostate cancer tissues. Cancer Res. 58: 204-209.

Suzuki, A., Itami, S., Ohishi, M., Hamada, K., Inoue, T., Komazawa, N., Senoo, H., Sasaki, T., Takeda, J., Manabe, M., et al. 2003. Keratinocyte-specific Pten deficiency results in epidermal hyperplasia, accelerated hair follicle morphogenesis and tumor formation. Cancer Res. 63: 674-681.

Tapon, N., Ito, N., Dickson, B.J., Treisman, J.E., and Hariharan, I.K. 2001. The Drosophila tuberous sclerosis complex gene homologs restrict cell growth and cell proliferation. Cell 105: 345-355.
You, M.J., Castrillon, D.H., Bastian, B.C., O’Hagan, R.C., Bosenberg, M.W., Parsons, R., Chin, L., and DePinho, R.A. 2002. Genetic analysis of Pten and Ink4a/Arf interactions in the suppression of tumorigenesis in mice. Proc. Natl. Acad. Sci. 99: $1455-1460$.

Zhang, H., Cicchetti, G., Onda, H., Koon, H.B., Asrican, K., Bajraszewski, N., Vazquez, F., Carpenter, C.L., and Kwiatkowski, D.J. 2003. Loss of Tsc1/Tsc2 activates mTOR and disrupts PI3K-Akt signaling through downregulation of PDGFR. J. Clin. Invest. 112: 1223-1233. 


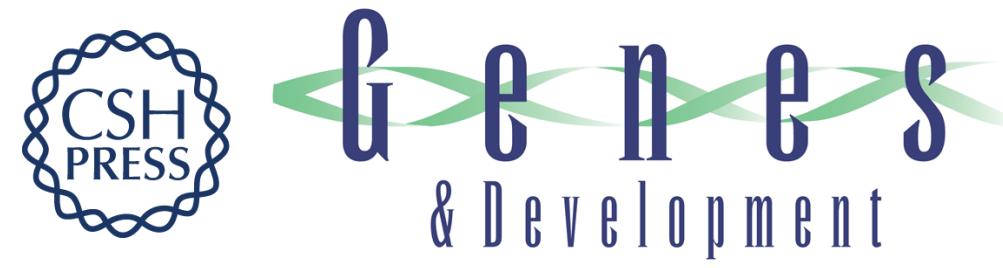

\section{Genetic analysis of Pten and Tsc2 functional interactions in the mouse reveals asymmetrical haploinsufficiency in tumor suppression}

Li Ma, Julie Teruya-Feldstein, Nille Behrendt, et al.

Genes Dev. 2005, 19:

Access the most recent version at doi:10.1101/gad.1314405

Supplemental
Material $\quad$ http://genesdev.cshlp.org/content/suppl/2005/07/18/gad.1314405.DC1

References This article cites 29 articles, 13 of which can be accessed free at:

http://genesdev.cshlp.org/content/19/15/1779.full.html\#ref-list-1

License

Email Alerting Receive free email alerts when new articles cite this article - sign up in the box at the top

Service right corner of the article or click here.

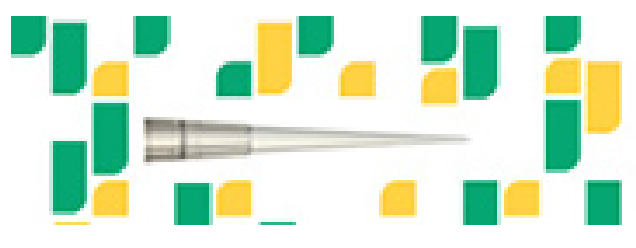

Focused on your science. 